\title{
Hybrid robust observers for locally Lipschitz systems
}

\author{
Alexey A. Bobtsov, Member, IEEE, Denis V. Efimov, Member, IEEE
}

\begin{abstract}
State observer design procedure is proposed for nonlinear locally Lipschitz systems. Possible presence of disturbances is taken into account. The solution is based on logic-based control approach applicable to nonlinear systems with bounded solutions.
\end{abstract}

\section{INTRODUCTION}

$\mathrm{T}_{\mathrm{s}}^{\mathrm{H}}$ HE state observers design problem for nonlinear systems has been an area of intensive research during the last two decades. There exists a lot of solutions in the area dealing with diverse forms of systems models. Among them it is necessary to mention high gain techniques [4], [9], [15]; sliding mode observers [7], [8], [31], [37]; nonlinear coordinate changes [5], [19], [20], [21]; numerical techniques [23]; approaches dealing with smooth [14], [34] and nonsmooth output functions [16]. Adaptive observers are designed for systems with parametric uncertainties [6], [12], [33], [35], [38]. Observers design procedures find their applications not only in areas of control under partial measurements [15], but also for fault detection [3], [13], [33], [36] systems synchronization [22] and secured data transmission and encoding [12], [24].

The class of Lipschitz nonlinear systems has seen much attention:

$$
\dot{\mathbf{x}}=\mathbf{A} \mathbf{x}+\varphi(\mathbf{y})+\mathbf{B} \mathbf{f}(\mathbf{x}, \mathbf{d}), \mathbf{y}=\mathbf{C} \mathbf{x},
$$

where $\mathbf{x} \in R^{n}$ is state vector; $\mathbf{d} \in R^{m}$ is disturbing input; $\mathbf{y} \in R^{P}$ is available for measurements output and functions $\boldsymbol{\varphi}: R^{p} \rightarrow R^{n}$ and $\mathbf{f}: R^{n+m} \rightarrow R^{p}$ are Lipschitz continuous (function $\mathbf{f}$ globally); constant matrices $\mathbf{A}, \mathbf{B}, \mathbf{C}$ have appropriate dimensions. An advance of this class of systems consists in fact, that almost all nonlinear systems of the form

$$
\dot{\mathbf{x}}=\mathbf{F}(\mathbf{x}, \mathbf{d}), \mathbf{y}=\mathbf{C} \mathbf{x},
$$

where $\mathbf{F}: R^{n+m} \rightarrow R^{n}$ is locally Lipschitz continuous, can be reduced to (1) at least locally.

Observers design for Lipschitz systems (1) was firstly

The first author is with Department of Control Systems and Informatics, Saint-Petersburg State University of Information Technologies Mechanics and Optics, Kronverksky av. 49, St. Petersburg, 197101, Russia, bobtsov@mail.ru. The second author is with CCS Lab., Institute for Problems of Mechanical Engineering, Bolshoi av., 61, V.O., St. Petersburg, 199178, Russia (the present place of work is Systems and Control@ Systems and Modeling Department of Electrical Engineering and Computer Science, B28 Université de Liège, B-4000 Liege Sart-Tilman, Belgium, efimov@montefiore.ulg.ac.be).

This paper presents research results of the Belgian Network DYSCO, funded by the Interuniversity Attraction Poles Programme, initiated by the Belgian State, Science Policy Office. The scientific responsibility rests with its author(s).and it is partly supported by RFBR grant 08-01-00775. considered in [32], where sufficient conditions ensuring asymptotic stability of the observer were proposed. In [27], [28] an iterative procedure based on algebraic Riccati equation solving was formulated. That result was later extended in [13]. A rather complete solution of these problems was presented in [29] and [30], where necessary and sufficient conditions directly related to observer matrices were proposed (a design procedure based on gradient optimization method was also presented in [29]). Robust properties of observers for (1) with respect to disturbing input $\mathbf{d}$ were analyzed in [1], [3], [25]. Adaptive observers design problem for Lipschitz systems was solved in [6], [35]. All these solutions were obtained under assumption on globality of Lipschitz property for function f. Under such assumption applying sufficiently high observer feedback gain it is possible to cancel influence of nonlinearity on observation error dynamics and the problem is solvable via linear systems approach.

Application of sliding mode approach allows one to ensure finite time convergence of a part of observation error to zero [7], [8], [31], [37]. Additionally, equivalent control methodic may help to estimate disturbances levels affecting on the system (that is useful for fault detection). Generically application of sliding mode approach for observation improves quality of transient processes and rate of error convergence.

If system is locally Lipschitz, only local solution is possible applying conventional approaches [28], [30], [32]. In [18], [39] attempts were carried out to find global solutions for special classes of systems (1) (without disturbances) based on tuned observer gains, which are continuously increasing while observation error are converging to zero. Development of this approach for generic case (enlarging class of systems or adding disturbances) meets obstacles dealing with possible unboundedness of observer feedback gains. In this work a solution of the problem is proposed for perturbed system (1), where growing observer gains are updated by discrete algorithm. On each step the new gains are substituted in the observer using logic-based scheme if the previous observer gains fail to satisfy some performance criteria. This prevents infinite growth of the gains in the case of disturbances presence.

The structure of the paper is as follows. Preliminary results are introduced in section 2. Main results are presented in section 3. Results of computer simulations are discussed in section 4 . 


\section{PRELIMINARIES}

The norm of Lebesgue measurable and essentially bounded function $\mathbf{d}: R_{+} \rightarrow R^{m}$ of time $t \geq 0$ will be defined as follows

$$
\|\mathbf{d}\|_{\left[t_{0}, t\right)}=\operatorname{ess} \sup \left\{|\mathbf{d}(t)|, t \in\left[t_{0}, t\right)\right\} .
$$

The set of all such functions with property $\|\mathbf{d}\|_{[0,+\infty)}=\|\mathbf{d}\|<+\infty$ we will further denote as $\mathcal{M}_{R^{m}}$. Norm $|\mathbf{R}|$ of matrix $\mathbf{R}$ is calculated as square root of its maximum singular value. As usual, continuous function $\sigma: R_{+} \rightarrow R_{+}$belongs to class $\mathcal{K}$ if it is strictly increasing and $\sigma(0)=0$; additionally it belongs to class $K_{\infty}$ if it is also radially unbounded; and continuous function $\beta: R_{+} \times R_{+} \rightarrow R_{+}$is from class $K \mathcal{L}$, if $\beta(\cdot, r) \in \mathcal{K}$ for any fixed $r \in R_{+}$, and $\beta(s, \cdot)$ is strictly decreasing to zero for any fixed $s \in R_{+}$.

If for all initial conditions $\mathbf{x}_{0} \in R^{n}$ and inputs $\mathbf{d} \in \mathcal{M}_{R^{m}}$ the solutions $\mathbf{x}\left(t, \mathbf{x}_{0}, \mathbf{d}\right)$ of the system (1) are defined for all $t \geq 0$, then the systems are called forward complete (necessary and sufficient conditions for a nonlinear system $\dot{\mathbf{x}}=\mathbf{F}(\mathbf{x}, \mathbf{d})$ to be forward complete can be found in [2]).

L e m m a 1 . Let $\mathbf{g}: R^{n} \rightarrow R^{l}$ be continuous and locally Lipschitz function, then there exist functions $\alpha_{1}, \alpha_{2} \in K$ such, that for all $\mathbf{x} \in R^{n}, \mathbf{z} \in R^{n}$ it holds:

$$
|\mathbf{g}(\mathbf{x})-\mathbf{g}(\mathbf{z})| \leq \alpha_{1}(1+|\mathbf{x}|) \alpha_{2}(1+|\mathbf{z}|)|\mathbf{x}-\mathbf{z}| .
$$

Proof. According to local Lipschitz property of function $\mathbf{g}$, for all $r>0, q>0$ there exists constant $L_{r, q}$ such, that

$$
|\mathbf{g}(\mathbf{x})-\mathbf{g}(\mathbf{z})| \leq L_{r, q}|\mathbf{x}-\mathbf{z}| \text { for }|\mathbf{x}| \leq r,|\mathbf{z}| \leq q .
$$

Consider function

$$
\gamma(r, q)=\sup _{|\mathbf{x}|=r,|\mathbf{z}|=q}|\mathbf{g}(\mathbf{x})-\mathbf{g}(\mathbf{z})| /|\mathbf{x}-\mathbf{z}|,
$$

which for any $r>0, q>0$ admits bounds $0<\gamma(r, q) \leq L_{r, q}$. Due to continuity of $\mathbf{g}$ there exists continuous function $a: R^{2} \rightarrow R_{+} \quad$ such, that $\gamma(r, q) \leq a(r, q)$. Therefore by standard arguments in this case there exist functions $\alpha_{1}, \alpha_{2} \in \mathcal{K}$ such, that the desired inequality holds.

The result of the lemma is somehow related with Lemma 2.5 from [18].

The next property is frequently used in adaptive control theory. Consider a linear dynamical system

$$
\dot{\xi}=\mathbf{A} \xi+\mathbf{B} v, \boldsymbol{\psi}=\mathbf{C} \xi
$$

with state $\xi \in R^{n}$, input $\boldsymbol{v} \in R^{p}$ and output $\boldsymbol{\psi} \in R^{p}$. Introduce the following notations:

$$
\delta(s)=\operatorname{det}\left(s \mathbf{I}_{n}-\mathbf{A}\right), W(s)=\mathbf{C}\left(s \mathbf{I}_{n}-\mathbf{A}\right)^{-1} \mathbf{B},
$$

$$
\varphi(s)=\delta(s) \operatorname{det}(W(s)), \Gamma=\lim _{s \rightarrow+\infty} s W(s),
$$

where $\mathbf{I}_{n}$ is identity matrix of size $n \times n$. Matrix inequalities are understood in sense of quadratic forms. System (2) is called minimum phase if the polynomial $\varphi(s)$ is Hurwitz (its zeros belong to the open left half-plane). System (2) is called hyper minimum phase if it is minimum phase and $\boldsymbol{\Gamma}=\boldsymbol{\Gamma}^{T}>0[10]$.

L e m ma 2 [11]. Let $\operatorname{rank}(\mathbf{B})=p$. Then the following properties are equivalent.

$1^{\circ}$. System (2) is hyper minimum phase.

$2^{\circ}$. There exist matrices $\mathbf{P}=\mathbf{P}^{T}>0$ and $\mathbf{K}$ of sizes $n \times n$ and $p \times p$ respectively such, that

$$
\mathbf{P}(\mathbf{A}+\mathbf{B K C})+(\mathbf{A}+\mathbf{B K C})^{T} \mathbf{P}<0, \mathbf{P B}=\mathbf{C}^{T} .
$$

$3^{\circ}$. There exist matrices $\mathbf{P}=\mathbf{P}^{T}>0$ and $\mathbf{K}$ of sizes $n \times n$ and $p \times n$ respectively such, that

$$
\mathbf{P}(\mathbf{A}+\mathbf{B K})+(\mathbf{A}+\mathbf{B K})^{T} \mathbf{P}<0, \mathbf{P B}=\mathbf{C}^{T} .
$$

$4^{\circ}$. There exist matrices $\mathbf{P}=\mathbf{P}^{T}>0$ and $\mathbf{K}$ of sizes $n \times n$ and $n \times p$ respectively such, that

$$
\mathbf{P}(\mathbf{A}-\mathbf{K C})+(\mathbf{A}-\mathbf{K C})^{T} \mathbf{P}<0, \mathbf{P B}=\mathbf{C}^{T} .
$$

\section{MAIN RESULTS}

In this work we suppose that the state vector $\mathbf{x}$ and the disturbing input $\mathbf{d}$ in the system (1) are bounded without precise information on their upper bounds.

A s s umption 1. Let for the system (1) $\|\mathbf{x}\|<+\infty$ and $\mathbf{d} \in \mathcal{M}_{R^{m}}$

The outline of this section is as follows. In the first part we introduce observer equations and substantiate the observer properties for the case when the exact upper estimates for $\|\mathbf{x}\|$ and $\|\mathbf{d}\|$ are given. In the second part a procedure is proposed, which is used for verification of accuracy for the given upper estimates for $\|\mathbf{x}\|$ and $\|\mathbf{d}\|$. In the third part the equations of the proposed hybrid state observer for the system (1) are presented and global convergence of estimation error is proven.

\subsection{Sliding mode state observer}

Consider the following robust state observer for (1):

$$
\dot{\mathbf{z}}=\mathbf{A} \mathbf{z}+\boldsymbol{\varphi}(\mathbf{y})+\mathbf{B}[\mathbf{f}(\mathbf{z}, 0)+\mathbf{u}]+\mathbf{K}(\mathbf{y}-\mathbf{C z}),
$$

where $\mathbf{z} \in R^{n}$ serves as vector $\mathbf{x}$ estimate; $\mathbf{K}$ is observer matrix gain with dimension $n \times p$, which value will be specified later; and $\mathbf{u} \in R^{p}$ is additional sliding mode feedback. Introduce into consideration dynamics of observation error $\mathbf{e}=\mathbf{x}-\mathbf{z}$ :

$$
\dot{\mathbf{e}}=[\mathbf{A}-\mathbf{K C}] \mathbf{e}+\mathbf{B}[\mathbf{f}(\mathbf{x}, \mathbf{d})-\mathbf{f}(\mathbf{z}, 0)-\mathbf{u}]
$$

and define 


$$
\mathbf{u}=\left\{\begin{array}{l}
k|\mathbf{C e}|^{-1} \mathbf{C e} \text { if } \mathbf{C e} \neq 0 \\
0 \text { otherwise }
\end{array}\right.
$$

where $k>0$ is design parameter to be calculated later.

From lemma 1 the following inequalities are satisfied for all $\mathbf{x} \in R^{n}, \mathbf{z} \in R^{n}, \mathbf{d} \in R^{m}$ :

$$
\begin{aligned}
& |\mathbf{f}(\mathbf{x}, \mathbf{d})-\mathbf{f}(\mathbf{z}, 0)| \leq \alpha_{1}(1+|(\mathbf{x}, \mathbf{d})|) \times \\
& \times \alpha_{2}(1+|\mathbf{z}|)|(\mathbf{x}, \mathbf{d})-(\mathbf{z}, 0)|
\end{aligned}
$$

for some functions $\alpha_{1}, \alpha_{2} \in \mathcal{K}$. For $\mathbf{d} \in \mathcal{M}_{R^{m}}$ the last inequality can be rewritten as follows:

$$
\begin{aligned}
& \alpha_{1}(1+|(\mathbf{x}, \mathbf{d})|) \alpha_{2}(1+|\mathbf{z}|)|(\mathbf{x}, \mathbf{d})-(\mathbf{z}, 0)| \leq \\
& \leq \alpha_{1}(1+|\mathbf{x}|+\|\mathbf{d}\|) \alpha_{2}(1+|\mathbf{z}|)[|\mathbf{x}-\mathbf{z}|+\|\mathbf{d}\|] .
\end{aligned}
$$

Denote

$$
\begin{gathered}
L(X, D, Z)=\alpha_{1}(1+X+D) \alpha_{2}(1+Z), \\
F(X, D, Z)=\alpha_{1}(1+X+D) \alpha_{2}(1+Z)[X+Z+D],
\end{gathered}
$$

then for all $|\mathbf{x}| \leq X,|\mathbf{z}| \leq Z$ and $\|\mathbf{d}\| \leq D$,

$$
\begin{gathered}
|\mathbf{f}(\mathbf{x}, \mathbf{d})-\mathbf{f}(\mathbf{z}, 0)| \leq L(X, D, Z)[|\mathbf{x}-\mathbf{z}|+D], \\
|\mathbf{f}(\mathbf{x}, \mathbf{d})-\mathbf{f}(\mathbf{z}, 0)| \leq F(X, D, Z) .
\end{gathered}
$$

As in conventional sliding mode observer approach [7], [8], [31], [37] the following property is required.

Assumption 2. The system (2) (where the matrices $\mathbf{A}, \mathbf{B}$ and $\mathbf{C}$ are from (1)) is hyper minimum phase.

Under this assumption according to lemma 2 there exist positive definite symmetric matrices $\mathbf{P}$ and $\mathbf{Q}$ such, that

$$
\mathbf{P}(\mathbf{A}-\mathbf{K C})+(\mathbf{A}-\mathbf{K C})^{T} \mathbf{P}=-\mathbf{Q}, \mathbf{P B}=\mathbf{C}^{T} .
$$

Consider for system (4), (5) Lyapunov function $V(\mathbf{e})=\mathbf{e}^{T} \mathbf{P} \mathbf{e}$, which time derivative has form:

$$
\begin{aligned}
& \dot{V}=\mathbf{e}^{T}\left[(\mathbf{A}-\mathbf{K C})^{T} \mathbf{P}+\mathbf{P}(\mathbf{A}-\mathbf{K C})\right] \mathbf{e}+ \\
& +2 \mathbf{e}^{T} \mathbf{P} \mathbf{B}[\mathbf{f}(\mathbf{x}, \mathbf{d})-\mathbf{f}(\mathbf{z}, 0)-\mathbf{u}]=-\mathbf{e}^{T} \mathbf{Q} \mathbf{e}+ \\
& +2 \mathbf{e}^{T} \mathbf{C}^{T}[\mathbf{f}(\mathbf{x}, \mathbf{d})-\mathbf{f}(\mathbf{z}, 0)-\mathbf{u}] .
\end{aligned}
$$

Assume that $\|\mathbf{x}\| \leq X_{0},\|\mathbf{d}\| \leq D_{0}$ and $\|\mathbf{z}\| \leq Z_{0}$ for some $X_{0}>0, D_{0}>0, Z_{0}>0$, i.e. additionally to assumption 1 suppose that upper estimates on the system (1) state and disturbances are known (constant $Z_{0}$ is always assigned by a designer), then we obtain:

$$
\dot{V} \leq-\mathbf{e}^{T} \mathbf{Q e}+2|\mathbf{C e}|\left[F\left(X_{0}, D_{0}, Z_{0}\right)-k\right] .
$$

In this case a natural choice is $k>F\left(X_{0}, D_{0}, Z_{0}\right)$, then

$$
\dot{V}(t) \leq-2 \alpha V(t), \alpha=0.5 \lambda_{\min }(\mathbf{Q}) / \lambda_{\max }(\mathbf{P}),
$$

where $\lambda_{\min }(\mathbf{Q}), \lambda_{\max }(\mathbf{P})$ are minimum and maximum eigenvalues of corresponding matrices, $t \geq t_{0} \geq 0$. Thus, observation error $\mathbf{e}$ admits the exponential estimate

$$
\begin{gathered}
\left(\left|\mathbf{e}\left(t_{0}\right)\right| \leq\left|\mathbf{z}\left(t_{0}\right)\right|+X_{0}, \rho=\sqrt{\lambda_{\max }(\mathbf{P}) / \lambda_{\min }(\mathbf{P})}\right): \\
|\mathbf{e}(t)| \leq \rho\left(X_{0}+Z_{0}\right) e^{-\alpha\left(t-t_{0}\right)}, t \geq t_{0} .
\end{gathered}
$$

Unfortunately the values $X_{0}, D_{0}$ are not known and in general case for particular $k$ the inequality $k>F\left(X_{0}, D_{0}, Z_{0}\right)$ can be violated. Therefore, it is necessary to propose a procedure for values $X_{0}, D_{0}$ validation. To do so, denote $\mathbf{e}_{y}=\mathbf{C e}$ and consider dynamics of this variable:

$$
\dot{\mathbf{e}}_{y}=\mathbf{C}[\mathbf{A}-\mathbf{K C}] \mathbf{e}+\mathbf{C B}\left[\mathbf{f}(\mathbf{x}, \mathbf{d})-\mathbf{f}(\mathbf{z}, 0)-k\left|\mathbf{e}_{y}\right|^{-1} \mathbf{e}_{y}\right] .
$$

Time derivative of auxiliary Lyapunov function $W\left(\mathbf{e}_{y}\right)=0.5 \mathbf{e}_{y}^{T} \mathbf{e}_{y}$ takes form:

$$
\begin{aligned}
\dot{W} & =\mathbf{e}_{y}^{T} \mathbf{C}[\mathbf{A}-\mathbf{K C}] \mathbf{e}+\mathbf{e}_{y}^{T} \mathbf{C B}[\mathbf{f}(\mathbf{x}, \mathbf{d})-\mathbf{f}(\mathbf{z}, 0)]- \\
& -k \mathbf{e}_{y}^{T} \mathbf{C B}\left|\mathbf{e}_{y}\right|^{-1} \mathbf{e}_{y} .
\end{aligned}
$$

If constants $X_{0}, D_{0}, Z_{0}$ are chosen high enough and properties $\|\mathbf{x}\| \leq X_{0},\|\mathbf{d}\| \leq D_{0},\|\mathbf{z}\| \leq Z_{0}$ are true, then

$\mathbf{e}_{y}^{T} \mathbf{C}[\mathbf{A}-\mathbf{K C}] \mathbf{e}+\mathbf{e}_{y}^{T} \mathbf{C B}[\mathbf{f}(\mathbf{x}, \mathbf{d})-\mathbf{f}(\mathbf{z}, 0)] \leq$

$\leq\left|\mathbf{e}_{y}\right|\{|\mathbf{C}[\mathbf{A}-\mathbf{K C}]\|\mathbf{e}|+| \mathbf{C B}\| \mathbf{f}(\mathbf{x}, \mathbf{d})-\mathbf{f}(\mathbf{z}, 0)|\} \leq$

$\leq\left|\mathbf{e}_{y}\right|\left\{|\mathbf{C}[\mathbf{A}-\mathbf{K C}] \| \mathbf{e}|+|\mathbf{C B}|\left[L\left(X_{0}, D_{0}, Z_{0}\right)|\mathbf{e}|+D_{0}\right]\right\}$

and $k \mathbf{e}_{y}^{T} \mathbf{C B}\left|\mathbf{e}_{y}\right|^{-1} \mathbf{e}_{y} \geq k \lambda_{\min }(\mathbf{C B})\left|\mathbf{e}_{y}\right|$ (matrix $\mathbf{C B}$ is positive definite and symmetric due to assumption 2 since $\left.\mathbf{B}^{T} \mathbf{P} \mathbf{B}=\mathbf{B}^{T} \mathbf{C}^{T}\right)$. Therefore,

$$
\begin{aligned}
\dot{W} \leq & \leq \mathbf{e}_{y} \mid\{|\mathbf{C}[\mathbf{A}-\mathbf{K C}] \| \mathbf{e}|+|\mathbf{C B}| \times \\
& \left.\times\left[L\left(X_{0}, D_{0}, Z_{0}\right)|\mathbf{e}|+D_{0}\right]-k \lambda_{\min }(\mathbf{C B})\right\} .
\end{aligned}
$$

If $k>F\left(X_{0}, D_{0}, Z_{0}\right)$ and the estimate (6) is satisfied, then for any $\varepsilon>0$ there exists time $T_{\varepsilon}\left(X_{0}, Z_{0}\right) \geq t_{0}$ such that $|\mathbf{e}(t)| \leq \varepsilon$ for $t \geq T_{\varepsilon}\left(X_{0}, Z_{0}\right)$, where

$$
T_{\varepsilon}(X, Z)=t_{0}-\alpha^{-1} \ln (\varepsilon /\{\rho(X+Z)\}) \text {. }
$$

Time $T_{\varepsilon}\left(X_{0}, Z_{0}\right)$ defines length of the time interval, which is required for system (3) to observe state $\mathbf{x}$ of system (1) with predefined accuracy $\varepsilon$. Let additionally

$$
\begin{aligned}
k \geq & \lambda_{\min }(\mathbf{C B})^{-1}\{1+|\mathbf{C}[\mathbf{A}-\mathbf{K} \mathbf{C}]| \varepsilon+ \\
& \left.+|\mathbf{C B}|\left[L\left(X_{0}, D_{0}, Z_{0}\right) \varepsilon+D_{0}\right]\right\},
\end{aligned}
$$

then for $t \geq T_{\mathcal{E}}\left(X_{0}, Z_{0}\right)$,

$$
\dot{W}(t) \leq-\left|\mathbf{e}_{y}\right|=-\sqrt{2} \sqrt{W(t)}
$$

and for $T_{\mathcal{E}}\left(X_{0}, Z_{0}\right) \leq t \leq T_{0}\left(X_{0}, Z_{0}\right)$

$$
\begin{gathered}
W(t) \leq 0.5\left(\left|\mathbf{e}_{y}\left(T_{\varepsilon}\left(X_{0}, Z_{0}\right)\right)\right|+T_{\varepsilon}\left(X_{0}, Z_{0}\right)-t\right)^{2} ; \\
W(t)=0, t \geq T_{0}\left(X_{0}, Z_{0}\right),
\end{gathered}
$$

where $\quad T_{0}\left(X_{0}, Z_{0}\right)=\left|\mathbf{e}_{y}\left(T_{\varepsilon}\left(X_{0}, Z_{0}\right)\right)\right|+T_{\varepsilon}\left(X_{0}, Z_{0}\right)$. Thus, the time $T_{0}\left(X_{0}, Z_{0}\right)$ can be used for detection of correctness of values $X_{0}, D_{0}, Z_{0}$ choice, since available for measurements signal $\mathbf{e}_{y}(t)=\mathbf{y}(t)-\mathbf{C z}(t)$ should possess the constrain $\mathbf{e}_{y}(t)=0$ for $t \geq T_{0}\left(X_{0}, Z_{0}\right)$.

\subsection{Observer gains failures verification}


3.3. Global robust hybrid observer for locally Lipschitz nonlinear systems

Assume that there exists $t^{\prime} \geq T_{0}\left(X_{0}, Z_{0}\right)$ such, that $\left|\mathbf{e}_{y}\left(t^{\prime}\right)\right|>0$, then it means that constants $X_{0}, D_{0}, Z_{0}$ have been chosen not sufficiently high. Taking for $X_{0}, D_{0}$ and $Z_{0}$ new higher values it is necessary to repeat all described above steps, which in general case can be formalized as follows:

$$
\begin{aligned}
& X_{i}=h_{x}\left(i, X_{i-1}\right), D_{i}=h_{d}\left(i, D_{i-1}\right), Z_{i}=h_{z}\left(i, Z_{i-1}\right) \\
& X_{0}>0, D_{0}>0, Z_{0}>|\mathbf{z}(0)| \text {, } \\
& i=1,2,3, \ldots N \leq+\infty \text {; } \\
& k_{i}>\max \left\{F\left(X_{i}, D_{i}, Z_{i}\right),\{1+|\mathbf{C}[\mathbf{A}-\mathbf{K} \mathbf{C}]| \varepsilon+\right. \\
& \left.\left.+|\mathbf{C B}|\left[L\left(X_{i}, D_{i}, Z_{i}\right) \varepsilon+D_{i}\right]\right\} / \lambda_{\min }(\mathbf{C B})\right\} ; \\
& \begin{aligned}
& \dot{\mathbf{z}}(t)=\operatorname{Proj}\left[\mathbf{z}, \mathbf{A} \mathbf{z}(t)+\boldsymbol{\varphi}(\mathbf{y}(t))+\mathbf{B} \mathbf{u}_{i}(t)+\right. \\
&\quad+\mathbf{B} \mathbf{f}(\mathbf{z}(t), 0)+\mathbf{K}(\mathbf{y}(t)-\mathbf{C} \mathbf{z}(t))],
\end{aligned} \\
& \mathbf{u}_{i}(t)=k_{i}|\mathbf{y}(t)-\mathbf{C} \mathbf{z}(t)|^{-1}[\mathbf{y}(t)-\mathbf{C} \mathbf{z}(t)] ;
\end{aligned}
$$

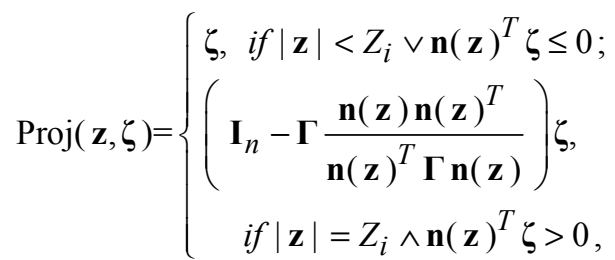

$$
\begin{aligned}
& t_{i+1}=\underset{t \geq T_{i}^{0}}{\operatorname{argsup}}\left\{\left|\mathbf{e}_{y}(t)\right|>0\right\}, t_{0}=0, \\
& t \geq T_{i}^{0} \\
& T_{i}^{0}=\left|\mathbf{e}_{y}\left(T_{i}^{\varepsilon}\right)\right|+T_{i}^{\varepsilon}, T_{i}^{\varepsilon}=t_{i}-\frac{1}{\alpha} \ln \left(\frac{\varepsilon}{\rho\left(X_{i}+Z_{i}\right)}\right),(12
\end{aligned}
$$

where discrete systems (7) have well defined strictly increasing solutions for any $X_{0}>0, D_{0}>0, Z_{0}>0$ for all $i \geq 1$, constant $\varepsilon>0$ and matrix $\Gamma>0$ can be taken arbitrary; $\mathbf{n}(\mathbf{z})$ is the unit outward normal vector for $|\mathbf{z}|=Z_{i}$, then projection algorithm (10) ensures existence and boundedness of the system (9) solutions [17], [26] for cases of wrong choices of values $X_{i}, D_{i}, Z_{i}$ (which are calculated from (7) at time instants $t_{i}$ ).

The following result describes stability properties of this hybrid observation algorithm.

$\mathrm{Th}$ e or e m 1. Let assumptions 1, 2 hold and discrete systems (7) have well defined strictly increasing to infinity solutions for any $X_{0}>0, D_{0}>0, Z_{0}>0$ for all $i \geq 1$.

Then for any $\varepsilon>0, \Gamma>0$ for the system (1) with the algorithm (7)-(12) it holds that

$-\|\mathbf{z}\|<+\infty$;

- there exists the last step $N<+\infty$ of the algorithm such, that $|\mathbf{y}(t)-\mathbf{C z}(t)|=0$ for all $t \geq T_{N}^{0}$;

- there exists $T_{N}^{\varepsilon} \leq T<+\infty$ such that

$$
|\mathbf{x}(t)-\mathbf{z}(t)| \leq \varepsilon \text { for all } t \geq T .
$$

Proof. The operation of algorithm (7)-(12) can be explained in the following way. For any $i \geq 1$ at time instant $t_{i}$ the values $X_{i}, D_{i}, Z_{i}$ are derived from equations (7) (for $i=0$ the initial conditions $X_{0}, D_{0}, Z_{0}$ are used). Further, the value of sliding feedback gain $k_{i}$ is calculated in accordance with (8). Finally, the time instants $T_{i}^{0}$ and $T_{i}^{\varepsilon}$ are calculated from (12). If constants $X_{i}, D_{i}, Z_{i}$ have been chosen correctly, then the value $T_{i}^{\varepsilon}$ defines the time instant after which the system insures the state observation with prescribed accuracy $\varepsilon$ (i.e. $|\mathbf{x}(t)-\mathbf{z}(t)| \leq \varepsilon$ for all $t \geq T_{i}^{\varepsilon}$ ). The value $T_{i}^{0}$ in this case indicates the time instant when available for measurements signal $\mathbf{e}_{y}(t)=\mathbf{y}(t)-\mathbf{C z}(t)$ reaches for zero. At this point "off-line" part of calculations in algorithm (7)-(12) is finished (it is assumed that all these computations are done at the time instant $t_{i}$ ) and "on-line" operations are initiated. During "on-line" part the observer (9) attempts to estimate the system (1) state vector values $\mathbf{x}(t)$. The presence of projection ensures boundedness of the vector $|\mathbf{z}(t)| \leq Z_{i}$ for $t \in\left[t_{i}, t_{i+1}\right)$ (the exceeding of bound $Z_{i}$ by vector $\mathbf{z}(t)$ norm indirectly means that some or all values from $X_{i}, D_{i}, Z_{i}$ have been chosen wrongly) and prevents finite time escapes for nonlinear perturbed system (9). Algorithm (9), (10) provides continuity of $\mathbf{z}(t)$ for $t \in\left[t_{i}, t_{i+1}\right.$ ) and, thus, for all $t \geq 0$ (the right hand side of the system is piecewise continuous vector function).

If for some $t^{\prime} \geq T_{i}^{0}$ the condition $\left|\mathbf{e}_{y}\left(t^{\prime}\right)\right|>0$ is satisfied, it implies that values $X_{i}, D_{i}, Z_{i}$ have been taken not sufficiently high. Then $t_{i+1}=t^{\prime}$ and it is necessary to repeat all steps of the algorithm. Since $\|\mathbf{x}\|<+\infty$ and $\|\mathbf{d}\|<+\infty$, for strictly increasing sequences $X_{i}, D_{i}, Z_{i}$ there exists an index $i=N<+\infty$ such, that $\|\mathbf{x}\|<X_{N},\|\mathbf{d}\|<D_{N}$. In this case it holds that $|\mathbf{e}(t)| \leq \varepsilon$ for all $t \geq T_{N}^{\varepsilon}$ and $\left|\mathbf{e}_{y}(t)\right|=0$ for $t \geq T_{N}^{0} ;|\mathbf{z}(t)| \leq Z_{N}$ for all $t \geq t_{N}$.

Finally note, that it may be the case that $\left|\mathbf{e}_{y}(t)\right|=0$ for all $t \geq T_{N}^{0}$, but $\|\mathbf{x}\| \geq X_{N}$ and/or $\|\mathbf{d}\| \geq D_{N}$. In this case due to assumption 2 the equality $\dot{V}=-\mathbf{e}^{T} \mathbf{Q e}$ holds, which ensures convergence of the observation error to desired neighborhood of the origin for some finite time $T \geq T_{N}^{\mathcal{\varepsilon}}$.

According to the result of Theorem 1 the observer (7)-(12) provides finite time convergence of observation error to $\varepsilon$-neighborhood of zero for any $\varepsilon>0$ for all initial conditions $\mathbf{x}_{0} \in R^{n}$ and $\mathbf{d} \in \mathcal{M}_{R^{m}}$ when the corresponding solutions are bounded (assumption 1 is satisfied). The main restrictions on class of admissible for the approach systems 
(1) are formulated in assumption 2. The linear part of the system (1) (i.e. the system (2)) has to be hyper minimum phase, that for instance implies relative degree 1 for the system (2). The lower estimate $T_{N}^{\varepsilon}$ on the time of convergence to the $\varepsilon$-neighborhood of zero for the error $\mathbf{e}$ is calculated during the algorithm processing.

R e mark 1. Let us stress that application of adaptive control approach for continuous tuning of the gain $k$ in sense of works [18], [39] is not possible here in general case. Indeed, the desired value for the gain $k$ can be defined by function $F(\|\mathbf{x}\|,\|\mathbf{d}\|,\|\mathbf{z}\|)$, where $\|\mathbf{x}\|$ and $\|\mathbf{d}\|$ are unknown constant, while $\|\mathbf{z}\|$ is the state of observer. Thus, the desired value $F(\|\mathbf{x}\|,\|\mathbf{d}\|,\|\mathbf{z}\|)$ is not a constant and depends in nonlinear fashion on $\mathbf{z}$. This obstruction comes from lemma 1 (Lipschitz constant of locally Lipschitz system nonlinearly depends on all arguments).

To resolve this "loop" logic-based scheme is used and discrete algorithms (7) are applied in this work.

Remark 2. Optimization of functions $h_{x}, h_{d}, h_{z}$ form can guarantee convergence of the algorithm with minimum number of steps or at least it can provide a desired upper estimate on number of the algorithm steps. For example, let $h_{x}\left(i, X_{i}\right)=\exp (\gamma i)$ for some $\gamma>0$, then $N=\operatorname{round}\left\{\gamma^{-1} \ln (\|\mathbf{x}\|)\right\}$, where round $\{\cdot\}$ is rounding-off operator to closest bigger integer number.

Remark 3 . According to (12) the time instants $T_{i}^{\varepsilon}$ may go to infinity rather fast with growing $X_{i}$ and $Z_{i}$, that increases required time for each step execution since

$$
t_{i+1} \geq T_{i}^{0}=\left|\mathbf{e}_{y}\left(T_{i}^{\varepsilon}\right)\right|+T_{i}^{\varepsilon} .
$$

Values of $\mathbf{e}_{y}\left(T_{i}^{\varepsilon}\right)$ may be small and the growth of time follows by $T_{i}^{\varepsilon}$. To overcome this obstacle and reduce rate of time $T_{i}^{\varepsilon}$ increasing one can use different $\varepsilon>0$ for each step, for instance, one can substitute in (8) and (12) $\varepsilon=\chi \rho\left(X_{i}+Z_{i}\right) \quad$ for some $0<\chi<1$, then $T_{i}^{\varepsilon}=t_{i}-\alpha^{-1} \ln (\chi)$ and for $\left|\mathbf{e}_{y}\left(T_{i}^{\varepsilon}\right)\right| \leq E, \quad i \geq I \geq 0$, $E>0$ the time required for each step $i \geq I$ execution becomes also bounded (in the case of explicit failure):

$$
t_{i+1} \geq T_{i}^{0}=t_{i}+E-\alpha^{-1} \ln (\chi) .
$$

Remark 4 . If for wrongly chosen constants $X_{i}, D_{i}$, $Z_{i}$ the vector $\mathbf{z}(t)$ at time instant $t_{i+1}$ belongs to its boundary $Z_{i}$ (or close to it) when it is worth to modify the algorithm and reset the value of vector $\mathbf{z}$, i.e. $\mathbf{z}\left(t_{i+1}\right)=\mathbf{z}\left(t_{0}\right)$ or $\mathbf{z}\left(t_{i+1}\right)=0$ for instances.

\section{Simulations}

Let us consider a second order nonlinear system

$$
\begin{gathered}
\mathbf{A}=[-12 ; 11], \mathbf{B}=[0 ; 1]^{T}, \mathbf{C}=\mathbf{B}^{T}, \\
\varphi(y)=0, f(\mathbf{x}, d)=-x_{1}^{3}+d,
\end{gathered}
$$

where matrix $\mathbf{A}$ is unstable, but the system has globally bounded solutions and it is oscillating (assumption 1 holds). Since $\varphi(s)=s+1$ and $\lim _{s \rightarrow+\infty} s W(s)=1$, the system is hyper minimum phase and assumption 2 is satisfied. According to theorem 1 the observer (7)-(12) has to ensure robust state estimation for any initial conditions and bounded disturbances. For $\mathbf{K}=[2 ; 2]^{T}$ we have $\mathbf{P}=\mathbf{I}_{2}$ and $\mathbf{Q}=[-21 ; 1-2], \alpha=\rho=1$. Let $k_{0}=1, d(t)=\sin (0.1 t)$, $X_{0}=D_{0}=Z_{0}=0.1, \varepsilon=0.1$ then for initial conditions $\mathbf{x}(0)=[1 ; 1]$ and $\mathbf{x}(0)=[2 ; 2]$ the corresponding graphics of the system (13) output are presented in Fig. 1, norms of observer error e are plotted in Fig. 2 and increasing gains of the observer $k_{i}$ are shown in Fig 3 (the former two in logarithmic scales), $0 \leq t \leq 200 \mathrm{sec}$.

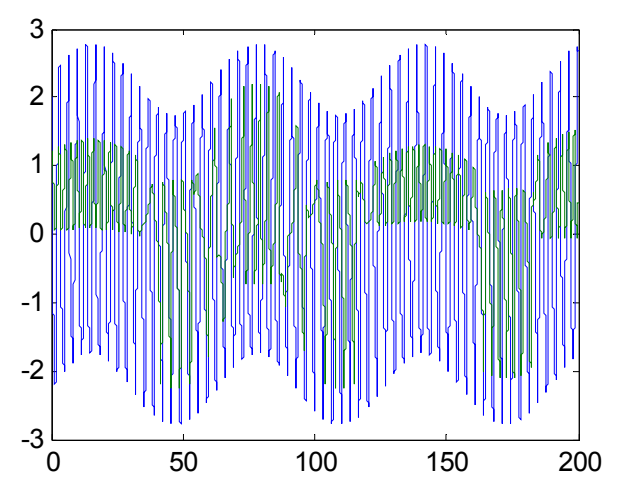

Fig. 1. The system (13) output.

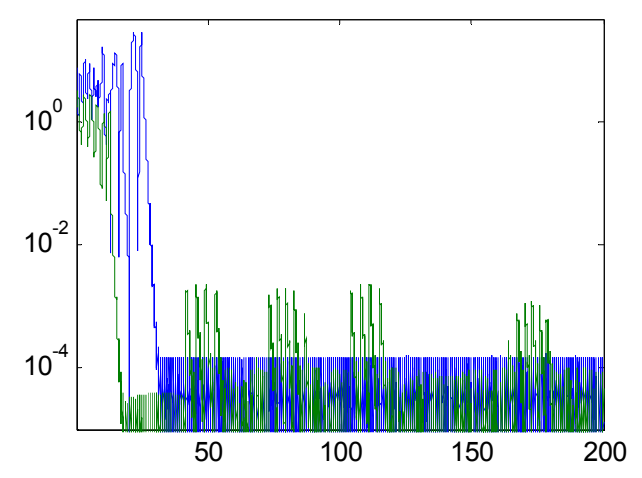

Fig. 2. Norms of observation error $\mathbf{e}$.

According to this simulation the observer gain quickly increases ensuring required quality of observation, while the output then demonstrates complex oscillations changing range and frequency. Asymptotical error fluctuations are proportional to accuracy of the simulation performed in MATLAB 7.0.1.

\section{CONCLUSION}

The procedure for hybrid state observer design for nonlinear locally Lipschitz systems is proposed. Possible 
presence of signal uncertainties is taken into account. The solution is based on logic-based control approach applicable to nonlinear systems with bounded solutions. Finite time convergence of the state estimation error to any neighborhood of the origin is guaranteed. Computer simulations confirm applicability and performance of the proposed observer.

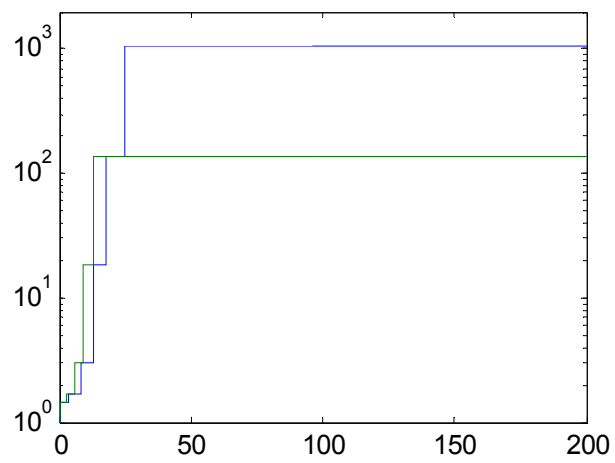

Fig. 3. Observer gains $k_{i}$.

\section{REFERENCES}

[1] Alessandri A. (2004). Observer design for nonlinear systems by using input-to-state stability. Proc. 43rd IEEE Conf. on Decision and Control, December 14-17, Atlantis, Paradise Island, Bahamas, pp. $3892-3897$.

[2] Angeli D., Sontag E.D. (1999). Forward completeness, unboundedness observability, and their Lyapunov characterizations. Systems and Control Letters, 38, pp. $209-217$.

[3] Besançon G. (2003). High-gain observation with disturbance attenuation and application to robust fault detection. Automatica, 39, pp. $1095-1102$.

[4] Busawon K., Farza M., Hammouri H. (1998). A simple observer for a Class of Nonlinear Systems. Appl. Math. Letters, 11(3), pp. 27-31.

[5] Ciccarella C., Dalla Mora M., Germani A. (1993). A Luenberger-like observer for nonlinear systems. Int. J. Control, 57, pp. 537-556.

[6] Cho Y.M., Rajamani R. (1997) A systematic approach to Adaptive Observer Synthesis for Nonlinear Systems. IEEE Trans. Autom. Control, 42(4), pp. 534-537.

[7] Edwards C., Spurgeon S. K. (1998). Sliding mode control: theory and applications. London: Taylor and Francis Ltd.

[8] Edwards C., Spurgeon S. K., Patton R. J. (2000). Sliding mode observers for fault detection and isolation. Automatica, 36, pp. 541553.

[9] Deza F., Bossanne D., Busvelle E., Gauthier J. P., Rakotopara D.(1993). Exponential observer for nonlinear systems. IEEE Trans. Autom. Control, 38(3), pp. 482-484.

[10] Efimov D.V., Fradkov A.L. (2006). Adaptive Tuning to Bifurcation for Time-Varying Nonlinear Systems. Automatica, 42, pp. 417-425.

[11] Fradkov A.L. (2003). Passification of nonsquare linear systems and feedback Yakubovich-Kalman-Popov Lemma. Europ. J. Contr., 6, pp. 573-582.

[12] Fradkov, A.L., Nikiforov V.O., Andrievsky B.R. (2002). Adaptive observers for nonlinear nonpassifiable systems with application to signal transmission. Proc. $41^{\text {th }}$ IEEE Conf. Decision and Control, Las Vegas, 10 -13 Dec., pp. 4706 - 4711.

[13] Garg V., Hedrick J. (1996). Fault detection filters for a class of nonlinear systems. Proc. Amer. Control Conf., Seattle, WA, Jun. 1996, pp. $1647-1651$.

[14] Johansson A., Medvedev A. (2003). An observer for systems with nonlinear output map. Automatica, 39(5), pp. 909-918.

[15] Khalil H.K. (2005). A note on the robustness of high-gain-observerbased controllers to unmodeled actuator and sensor dynamics. Automatica, 41, pp. 1821-1824.
[16] Kreisselmeier G., Engel R. (2003). Nonlinear observers for autonomous Lipschitz continuous systems. IEEE Trans. Autom. Control, 48(3), pp. 397-401.

[17] Krstić M., Kanellakopoulos I., Kokotović P.V. (1995). Nonlinear and adaptive control design. New York Wiley.

[18] Lei H., Wei J., Lin W. (2005). A global observer for observable autonomous systems with bounded solution trajectories. Proc. 44rd IEEE Conf. on Decision and Control, December 12-15, 2005, Sevilla, Spain, pp. $1911-1916$.

[19] Marino R. (1990). Adaptive observers for single-ouput nonlinear systems. IEEE Trans. Autom. Control, 35(9), pp. 1054-1058.

[20] Marino R., Tomei P. (1992). Adaptive observers for a class of multioutput nonlinear systems. Int. J. Adaptive Contr. Signal Processing, 6 pp. 353-365.

[21] Nijmeijer H., Fossen T.I. (1999). New Directions in Nonlinear Observer Design. London, U.K.: Springer-Verlag.

[22] Nijmeijer H., Mareels I.M.Y. (1997). An Observer Looks at Synchronization. IEEE Trans. Circuit Syst. I, 44(10), pp. 882 - 890.

[23] Nikoukhah R. (1998). A new methodology for observer design and implementation. IEEE Trans. Autom. Control, 43(2), pp. 229-234.

[24] Pecora L. and T. Carrol (1990). Synchronization in chaotic systems, Phys.Rev. Lett., 64, pp. $821-824$.

[25] Pertew A.M., Marquez H.J., Zhao Q. (2006). Hinf Observer Design for Lipschitz Nonlinear Systems. IEEE Trans. Autom. Control, 51(7), pp. 1211-1216.

[26] Pomet J.-B., Praly L. (1992). Adaptive nonlinear regulation: Estimation from the Lyapunov equation. IEEE Trans. Automat. Contr., 37, pp. 729-740.

[27] Raghavan S. (1992). Observers and compensators for nonlinear systems with application to flexible joint robots. Ph.D. dissertation, Univ. California, Berkeley, CA.

[28] Raghavan S., Hedrick J. (1994). Observer design for a class of nonlinear systems. Int. J. Control, 59, pp. 515-528.

[29] Rajamani R. (1993). Observers for nonlinear systems with application to active automotive suspensions. Ph.D. dissertation, Univ. California, Berkeley, CA.

[30] Rajamani R. (1998). Observers for Lipschitz nonlinear systems. IEEE Trans. Autom. Control, 43(3), pp. 397-401.

[31] Tan C.P., F. Crusca, Aldeen M. (2008). Extended results on robust state estimation and fault detection. Automatica, 44, pp. 2027-2033.

[32] Thau F.E. (1973). Observing the state of non-linear dynamic systems. Int. J. Control, 17(3), pp. 471-479.

[33] Wang H., Huang Z.J., Daley S. (1997). On the use of adaptive updating rules for actuator and sensor fault diagnosis. Automatica, 33(2), pp. 217-225.

[34] Xia X., Zeitz M. (1997). On nonlinear continuous observers. Int. J. Control, 66(6), pp. 943-954.

[35] Xu A., Zhang Q. (2004). Nonlinear system fault diagnosis based on adaptive estimation. Automatica, 40, pp. 1181-1193.

[36] Xu A., Zhang Q. (2004). Residual Generation for Fault Diagnosis in Linear Time-Varying Systems. IEEE Trans. Autom. Control, 49(5), pp. 767-772.

[37] Yan X.-G., Edwards C. (2007). Nonlinear robust fault reconstruction and estimation using a sliding mode observer. Automatica, 43, pp. $1605-1614$

[38] Zhang, Q. (2002). Adaptive observer for multiple-input-multipleoutput (MIMO) linear time varying systems. IEEE Trans. Autom. Control, 47(3), pp. 525-529.

[39] Zhou J., Lu J., Lü J. (2006). Adaptive Synchronization of an Uncertain Complex Dynamical Network. IEEE Trans. Autom. Control, 51(4), pp. 652-656. 